Forthcoming in Inquiry; accepted version available at

https://doi.org/10.1080/0020174X.2021.1982404

\title{
Plural Metaphysical Supervaluationism
}

Cristian Mariani (Institut Néel, CNRS), Robert Michels (eidos, Università

della Svizzera italiana \& University of Bern), Giuliano Torrengo (University

of Milan \& Autonomous University of Barcelona)

\begin{abstract}
.
It has been argued that quantum mechanics forces us to accept the existence of metaphysical, mind-independent indeterminacy. In this paper we provide an interpretation of the indeterminacy involved in the quantum phenomena in terms of a view that we call Plural Metaphysical Supervaluationism. According to it, quantum indeterminacy is captured in terms of an irreducibly plural relation between the actual world and various misrepresentations of it.
\end{abstract}

\section{Keywords.}

Metaphysical Indeterminacy. Metaphysical Supervaluationism. Quantum Mechanics.

\section{Introduction}

There is a growing literature in philosophy discussing whether and how we can make sense of metaphysical indeterminacy (MI for short) or, as the received view has it, whether all indeterminacy is semantic. ${ }^{1}$ A question which has taken central stage in this discussion is whether quantum mechanics entails the existence of MI. This issue is particularly important, since if it does, this appears to give us broadly naturalistic reasons to abandon the received view and accept the existence of MI.

In this paper we develop a new view of MI, which we call Plural Metaphysical Supervaluationism. In $\S 2$ we discuss quantum mechanical

\footnotetext{
${ }^{1}$ The debate originates with Evans 1978. See e.g. Akiba \& Abasnezhad 2014. See also Rosen \& Smith 2004 and Barnes 2010. The received view is well exemplified by Lewis's claim that "[t]he only intelligible account of vagueness locates it in our thought and language" (1986, p. 212).
} 
indeterminacy. In $\S 3$ we introduce the core ideas of metaphysical supervaluationism, an account of MI due to Barnes \& Williams. $\S 4$ is dedicated to the development of our own account, which departs from metaphysical supervaluationism in several ways. In $\S 5$, we argue that plural metaphysical supervaluationism accommodates the quantum case better than its rivals.

\section{Quantum Indeterminacy}

Quantum indeterminacy (QI) emerges from the peculiar structure of property attribution to microscopic systems such as subatomic particles. It is well known that certain pairs of properties, the so-called incompatible ones, cannot jointly be assigned definite values. For instance, if we were to measure the observable position, the mathematical structure of the theory would prohibit the assignment of a definite value for momentum to the same system at the same time. This is because position and momentum are incompatible observables. Since the very birth of the theory, this peculiar feature of the microscopic world, which we shall call Lack of Value Definiteness (LVD for short), was taken to suggest that the world itself, not our representations of it, might sometimes lack determination. ${ }^{2}$

To understand what LVD consists of, let us consider a simple example from quantum mechanics (QM). Take the property known as spin. Roughly, a way to visualize it is by thinking of classical angular momentum. If microscopic particles were billiard balls, their spin would be the direction along which they rotate on a certain axis. Unlike classical angular momentum, spin is a quantized quantity, which means it is discrete. Thus, for each given axis, electrons (which are fermions, and so have spin-1/2) can only take two possible values, which we call up and down. It is an empirical fact that spin components in three mutually orthogonal directions (call them $x y$ and $z$ ) are incompatible observables. That is, if we know, say, that the electron $e$ has $\operatorname{spin}_{\text {Down }}$ on the $x$-axis (suppose we have measured it), we also know that its spin on the other two axis cannot be definitely assigned. We could use bra-ket notation to write the quantum states of the electron as follows:

\footnotetext{
${ }^{2}$ Heisenberg has often suggested a similar view (see the collection of papers from 2007). Schrödinger (1935) considers this option (the fifth section of the 'cat-experiment' paper is entitled 'Are the variables really blurred?'), but eventually rejects it.
} 


$$
\begin{aligned}
& e=\mid \downarrow x> \\
& e=|\downarrow x>=1 / \sqrt{ } 2| \downarrow z>+1 / \sqrt{ } 2 \mid \uparrow z> \\
& e=|\downarrow x>=1 / \sqrt{ } 2| \downarrow y>+1 / \sqrt{ } 2 \mid \uparrow y>
\end{aligned}
$$

(1) expresses the fact that the electron is in an eigenstate of having the value 'down' for the observable $x$-spin, while (2)-(3) express superposition states of the observables that are incompatible with $x$-spin, namely $z$-spin and $y$-spin. One major interpretative issue in quantum mechanics is related to what we should say about states of superposition like the above. If a system is in superposition of a certain observable, this seems to suggest that it does not possess a definite value (it is neither 'this' nor 'that', although 'being this' and 'being that' exhaust the possibilities). How are we supposed to make sense of this?

The first, quite natural reaction is to stress that everything we just said merely indicates our epistemic limitations-we just happen not to know which value is possessed by the system, yet it does always possess one. However, many foundational results in quantum theory, most crucially the Kochen-Specker theorem (1967), have shown that supplementing the theory with hidden variables (roughly: the values we have no access to) will not be possible in some cases. Superposition states need to be taken seriously from a metaphysical perspective, and positing metaphysical indeterminacy is one way of doing so.

Let us get back to LVD. Since to every eigenstate there are some corresponding superposition states, and since being in an eigenstate simply means possessing a definite-valued property, LVD in quantum mechanics is pervasive. To make a generalized statement, we need to look at how in QM physical properties are attributed to systems, a task that is performed by the so-called Eigenstate-Eigenvalue Link (EEL):

EEL. A quantum system $s$ has a definite value $v$ for the observable $O$ iff it is in an eigenstate of $O$ having eigenvalue $v$.

From EEL, we can straightforwardly derive that systems that are not in an eigenstate of having value $v$ for the corresponding properties, do not possess 
a definite value for those properties. And since, as we said earlier, to each eigenstate there always correspond certains superposition states, QM along with EEL describe a world in which properties are often instantiated indefinitely. This is, in a nutshell, LVD in quantum mechanics.

EEL is at the core of the standard interpretation of the theory, sometimes called Orthodox or Copenhagen QM. In the discussion about QI it is common to assume EEL as a good starting point, so as to give what Wallace calls an "interpretation-neutral discussion of the ontology of QM" (2016). By referring to EEL, for instance, Calosi \& Wilson (2019) distinguish three sources of QI: incompatible observables, superposition, entanglement. Each of these phenomena, once coupled with the EEL, will entail that the relevant systems lack value definitedness.

So the story goes for Orthodox QM. However, as it is well known, this approach is not the only way to interpret QM, and more importantly, due to the measurement problem it is not even a viable one according to numerous commentators (e.g. Maudlin 1995). Furthermore, Orthodox QM cannot be straightforwardly taken as realist with respect to the quantum state; but then, why should we take metaphysical lessons from a theory that cannot be taken at aiming at describing reality? In effect, in the recent debate on quantum indeterminacy there has been a substantial shift from a discussion of LVD in Orthodox QM (Skow 2010, Darby 2010, Bokulich 2014) towards a discussion within realist interpretations of the theory (Glick 2017, Calosi \& Wilson 2019, Calosi \& Mariani 2020; for an overview of the debate, see Calosi \& Mariani 2021) —where by realist we mean a certain attitude towards either the quantum state (see Miller 2014) or the observables (Rovelli 1996).

According to the major realist interpretations, the EEL is not the correct way to ascribe properties on the ground of the quantum state, so what happens to LVD? Glick (2017) and Chen (2020) both argue that once a precise underlying ontology is given to QM, any indeterminacy disappears. Here is Glick (2017):

If $[\ldots]$ one took the properties to be ontologically derivative and quantum states to be fundamental, there would be little room for metaphysical indeterminacy [...] any indeterminacy would occur at the non-fundamental level and hence may be viewed as eliminable. (p. 206, italics added)

Similarly Chen (2020): 
[...] we now have realist theories of quantum mechanics such as Bohm's theory, GRW collapse theory, and Everett's theory that make quantum mechanics precise. In those precise theories, ontic vagueness disappears: there is no indeterminacy in the fundamental ontology or fundamental dynamics. (12, italics added)

Even granting that there is no fundamental indeterminacy in the major approaches to QM, we should wonder whether Chen and Glick have successfully established that eliminativism is the right position to adopt - that is, whether we should understand all indeterminacy in non-metaphysical (either semantic or epistemic) terms. Another hypothesis is that the indeterminacy is still metaphysical, although derivative. After all, why expect that non-fundamental indeterminacy be necessarily semantic or epistemic? Indeed, Glick himself seems to maintain that indeterminacy affects the derivative ontology (see quote above).

Thus, LVD arguably affects the ontology of many realist approaches to QM - at least at some derivative level — as well as the fundamental ontology of Orthodox QM (granting that we could make sense of it) ${ }^{3}$. This strongly suggests that quantum indeterminacy has to be taken seriously, and that a clear metaphysical picture explaining this phenomenon has to be developed.

Supervaluationism is sometimes called the standard theory of vagueness (Varzi 2007, p. 633) and it arguably can also lay claim to this title in the literature on metaphysical indeterminacy. Metaphysical versions of the theory have been developed and defended by Akiba (see Akiba 2000a, 2004) and by Barnes and Williams in a series of papers (2011 contains the most mature version). However, it has been argued that the metaphysical version of supervaluationism is unable to account for QI (Darby 2010, Skow 2010, Calosi \& Wilson 2019). In the next section, we discuss metaphysical supervaluationism. In the rest of the paper we develop our theory, Plural Metaphysical Supervaluationism, discuss Metaphysical Supervaluationism's difficult relationship with quantum mechanics and argue that-unlike Metaphysical Supervaluationism—our theory can successfully capture QI.

\section{Metaphysical Supervaluationism}

\footnotetext{
${ }^{3}$ Bokulich (2014, p. 460), for instance, is explicit that an understanding of QI might provide good grounds for interpreting orthodox QM in a realist fashion.
} 
The fundamental idea of metaphysical supervaluationism is that MI can be modeled as indeterminacy with respect to which metaphysical precisifications of the actual world within a range of admissible ones correctly represents it. In this section, we will first introduce how the idea is developed by Barnes and Williams, ${ }^{4}$ and then give a rationale for why one should adopt a supervaluationist theory instead of one of the rival theories of MI which have been proposed in the literature.

\subsection{The Core Ideas of Barnes \& Williams'Version of Supervaluationism}

Barnes and Williams' (B\&W) theory is modeled on the supervaluationist theory of vagueness. Semantic vagueness is a particular sort of linguistic indeterminacy which affects predicates and it is generally associated with the Sorites paradox. A characteristic feature of vague predicates is that they do not allow us to draw a sharp line between objects to which they definitely apply and objects to which they definitely fail to apply. Consider for example a language $L$ that contains a predicate such as 'bald'. There are some people to which the predicate definitely applies (think of a person with absolutely no hairs on their head) and others to which it definitely fails to apply (think of a person with a full head of hair). But then there are also some people who are neither definitely bald, nor definitely not bald (think of someone who is in the process of balding, but still has a significant amount of hair). ${ }^{5}$

Supervaluationism appeals to variations of the language L (or variations of its interpretation, depending on the details of the account), in which it introduces a sharp delineation between the bald and the non-bald by fiat. If we do this for each vague predicate of a language, the result will be a precisification of $\mathrm{L}-\mathrm{a}$ complete classical variant (or interpretation) of $\mathrm{L}$. In its semantic understanding, supervaluationism is based on the idea that languages containing vague predicates always admit a multitude of admissible precisifications - the ones that are not ruled out by established conventions governing the meaning of the expressions of the language. The

\footnotetext{
${ }^{4}$ See in particular Barnes 2010, Barnes and Williams 2011, Barnes 2013, Williams 2008. We take the version developed in Barnes and Williams 2011 to be the definite version of their theory.

${ }^{5}$ This feature of vague predicates renders them susceptible to the sorites paradox, to which supervaluationism offers a solution. See Fine 1975, Keefe 2000, §7-8 for influential defenses of the theory.
} 
core idea of semantic supervaluationism is thus that vagueness can be understood as unsettledness regarding which of these admissible precisifications gives us (the correct interpretation of) the language.

Metaphysical Supervaluationism (MS) is an attempt to give a metaphysical construal of this idea. While in the case of semantic indeterminacy the precisification are variations of the language (or its interpretation) that contains vague expressions, in the case of MI, the precisifications are taken to be variations of the actual world. Unsettledness, thus, does not concern the semantic facts that govern the (vague) expressions; rather, it is unsettled which presification corresponds to the actual world.

Let us unpack this claim. The three concepts which need explaining here are that of unsettledness, that of a precisification of the actual world, and that of correspondence between precisifications and the actual world.

We will follow the approach to unsettledness taken in B\&W (2011); according to them, the notion expressed by "unsettled" in our schematic statement of the idea should be replaced by a pre-theoretical notion of indefiniteness which is not further analysable (2011, 108ff.). This means that the account of MI on offer in both B\&W's variant of MS and in ours is non-reductive in the sense that it relies on a primitive notion of indeterminacy, which cannot be explained in terms of other elements of more fundamental ontology.

We will also follow $\mathrm{B} \& \mathrm{~W}$ in identifying precisifications of the actual world with ersatz possible worlds which stand in a particular relation to it, such that they qualify as representations of it $(2011,114$.) In contrast to the possible worlds posited by modal realism which are exactly the same sort of maximally connected (analogous) spatiotemporal wholes as the actual world (cf. Lewis 1986), ersatz worlds are abstract entities. For the purposes of illustration it might help to think of them as maximally consistent sets of propositions, but we can remain neutral regarding questions of what sort of entities ersatz worlds are, as long as they are fit to play their theoretical role. Following usage, if an ersatz world $w$ contains a proposition $\mathrm{p}$, we will say that $\mathrm{p}$ is true in $\mathrm{w}$ - that is, $\mathrm{p}$ is true in a given possible (ersatz) world, or in a given precisification.

What does it take for an ersatz world to be a precisification of the actual world? B\&W's idea is that the precisifications of the actual world are those ersatz worlds which do not determinately misrepresent reality, i.e. the actual 
world $(2011,115)$. Note that ersatz worlds are meant to be complete representations of our universe, regardless of whether they are correct or incorrect ones. This will become important later on, when we will complicate our picture of the representational role of ersatz worlds in our theory.

This leaves the third notion, that of correspondence between the actual world and an ersatz world. Since unlike the actual world, ersatz worlds are abstract entities, correspondence can here not simply mean identity. However, leaving MI aside, one of the ersatz worlds can be actualized, which means that it is the one ersatz world which correctly represents the actual facts.

Putting these notions back together, we get the following core claim of B\&W theory:

$\mathbf{B} \& \mathbf{W}$. It is metaphysically indeterminate whether $p$ if, and only if, there is an ersatz world $\mathrm{w}$ which is a precisification of the actual world in which $p$ is true and an ersatz world w' which is a precisification of the actual world in which $\sim p$ is true and it is indefinite which of $\mathrm{w}$ and $\mathrm{w}$ ' is the actualized ersatz world.

\subsection{Alternatives to and a Rationale for MS}

$\mathrm{B} \& \mathrm{~W}$ 's theory is not the only theory of metaphysical indeterminacy on the market. Its three main rivals are: (i) the fuzzy logic-based theory developed in Rosen \& Smith (2004), which is based on an ontology of fuzzy properties; (ii) Wilson's determinable-based theory (2013, Calosi \& Wilson 2019), according to which metaphysical indeterminacy is to be understood in terms of states of affairs which are indeterminate in virtue of containing an object which has a determinable property, but either lacks or has multiple determinates of this determinable property at the same level of determination; and (iii) Akiba's (2000a, 2004) variant of MS, which is based on the idea that the world has, besides spatial and (perhaps) temporal and modal dimensions, also a precisificational dimension throughout which material objects extend.

There are two reasons which a priori speak for B\&W's theory. First, the fact that it builds on a supervaluationst framework means that it is in line with a potential unified explanation of a range of different kinds of indeterminacy in terms of the same underlying formal structure-including 
predicate vagueness (see Fine 1975, Keefe 2000), indeterminacy in mathematics (see Akiba 2000b, Field 1994), empty names treated as lacking determinate reference (van Fraassen 1966), vague identity statements (Varzi 2020), and the open future (Thomason 1970, Barnes \& Cameron 2008). A second reason is that B\&W's theory is metaphysically less committal than both Rosen \& Smith's theory, which posits fuzzy properties, and Wilson's, which requires both the existence of states of affairs and the brute, unexplained distinction between determinable and determinate properties. This second reason also speaks against Akiba's version of MS, since Akiba's reliance on a precisificational dimension is metaphysically more revisionary than B\&W's reliance on a machinery of ersatz worlds.

We take those to be good reasons to exploit MS to account for MI. However, B\&W's version of the view has certain drawbacks which we tackle in $\S 5$, after developing our view.

\section{$4 \quad$ Plural Metaphysical Supervaluationism}

\subsection{The Core Idea of PMS}

The main point of this section is to introduce a novel version of MS, which we call Plural Metaphysical Supervaluationism (PMS). The core difference between our version of MS and that of Barnes and Williams is that it takes the relation which holds between reality (the actual world, our universe, as opposed to the abstract ersatz worlds) and those ersatz worlds which qualify as precisifications of reality in a model to be a plural relation. B\&W take this relation, call it $R_{p}$, to be a singular relation which holds between reality and one ersatz world. Our proposal is to replace this singular relation by a relation which relates reality to a plurality of worlds. This means that while in B\&W's theory only single ersatz world can be precisifications, in ours it will usually be pluralities of ersatz words which constitute precisifications of the actual world. ${ }^{6}$

Importantly, we claim that the plurality of these worlds is irreducibly plural in at least some cases of MI. As we will discuss in detail in $\S 5$, these cases in particular include cases of MI which arise from quantum

\footnotetext{
${ }^{6}$ Note that we will later qualify the notion of precisification by distinguishing between the relations of admissibility, which holds between a possible universe and a model, and of co-precisification, which holds between ersatz worlds in a model and which corresponds to the accessibility relation in standard modal logic. See $\S$ 4.2.1.
} 
mechanics, i.e. cases of QI. In this section, we focus on developing the core idea of our theory in a general manner.

Irreducibly plural instantiations of properties are properties which are instantiated by a plurality of objects $x x,{ }^{7}$ but neither by any of the single objects among the $x x$, nor by any sub-plurality of the $x x$, i.e. any plurality consisting of some objects among the $x x$, but not of all of them. There is in general nothing unusual about such cases. Think for example of the relational property of holding hands to form a human chain around the base of the Empire State Building. We can imagine a group of protesters which forms a very tight chain around the building, so that if any of them were to leave, the remaining protesters could not hold hands to close the chain again. ${ }^{8}$ We claim that the relation of being a precisification of reality, $R_{p p}$, as applied to a plurality of ersatz worlds in particular cases of MI is of this kind: in such cases, each of the worlds contributes to precisifying reality, but none of them alone counts as a precisification of reality, and neither do just some, but not all of them.

So consider a case in which reality is metaphysically indeterminate regarding whether $p$ is the case. In this case, there are two ersatz worlds which correctly represent all the actual precise facts, but disagree regarding $p$. One of the two will represent reality as being such that $p$, the other as being such that $\sim p$. According to our view there are particular cases of MI of this sort in which, metaphorically speaking, both of them have to work together to count as precisifications of reality and none of the two alone does. This is the basic idea of PMS.

Our reliance on genuinely plural instances of a relation might appear problematic in cases in which there is no MI in reality. Similarly to semantic supervaluationism, PMS will model these cases as involving only a single ersatz world which does not definitely misrepresent reality rather than a plurality of ersatz worlds which do. This might seem to pose a problem, given that $R_{p p}$ is a plural relation which holds between reality and a plurality of ersatz-worlds. But this problem is only apparent. It is standard in plural logic to accept single objects as limiting cases of pluralities. ${ }^{9}$ Accordingly,

\footnotetext{
${ }^{7}$ We use double lowercase letters to indicate that a constant or variable is plural, as is standard in plural logic. See e.g. Oliver \& Smiley 2016.

${ }^{8}$ Contrast this to a case in which the protesters form a less tight chain, so that one or two of them could leave without breaking it.

${ }^{9}$ See Oliver \& Smiley 2016, p. 212.
} 
in a case where there is no MI, our theory will account for this in terms of there being a model containing a single world which does not definitely misrepresent reality. Similarly, our theory can fully accommodate cases of MI in which some or even each precisifications counts as a precisification of reality on its own. In such cases, the plurality is still a plurality, just not an irreducible one.

The move to the plural precisification-relation $R_{p p}{ }^{10}$ has several consequences for the overall theory. One consequence concerns the intuitive interpretation of what it means to count as a precisification, as already hinted at. Barnes and Williams characterize their relation $R_{p}$ as holding between reality and an ersatz world which "does not determinately misrepresent reality" $(2011,115)$. One might adapt this characterization to the plural relation $R_{p p}$ by simply describing it as the relation which holds between reality and a plurality of ersatz worlds which only taken together as a complete plurality are such that they do not determinately misrepresent reality. The idea would be that in cases of MI which require an irreducibly plural notion of precisification, a single ersatz world on its own always determinately misrepresents reality. To foreshadow the detailed discussion of QI of $\S 5$, in cases of QI, single ersatz-worlds assign precise values to properties where physics tells us that no definite value is had, and as a result, no single ersatz world can on its own count as an admissible precisification of reality. If we rely on a plurality of worlds instead to model QI, the same problem does not arise, since taken together, these worlds will not assign a definite value to the relevant property. The plurality gives us a range of different values which the property may take, of which we can single out none as the one correct value assigned to the property. So what the worlds together manage to do is to indicate exactly the respect in which reality is indeterminate. In the QI case, this means that there is a property (observable) to which no definite value is assigned. What they do not do is to represent reality as being such that the relevant observable has a particular value, or more generally speaking, as being such that the indeterminate portion of reality is as any single one among them tells us.

This is one option, but there is another, which we will rely on in what follows. We might instead characterize the relation as that of holding between reality and a plurality of ersatz worlds which only taken together as

\footnotetext{
${ }^{10}$ Note that we will discuss the aptness of this label for $R_{p p}$ in light of its role with respect to the model theory in $\S 3.2 .1$.
} 
a complete plurality are such that they do give us a precise representation of the indeterminacy of reality. While the first interpretation gives us, to use a term used by Calosi \& Wilson (2019), a gappy view of MI, since the worlds in the precisification collectively fail to determinately misrepresent reality, the second characterization gives us a glutty view of MI, since the plurality of worlds specify a local glut of logically possible ${ }^{11}$ states of affairs, ${ }^{12}$ none of which obtains. ${ }^{13}$ This glut of states of affairs provides a precise model of the complete state of our universe, including in particular the aspect, or aspects, with respect to which it is indeterminate.

The resulting model is precise in two ways. First, it precisely locates the indeterminacy in a particular (set of) states of affairs. Second, it also indicates precisely which variations of this state of affairs could possibly obtain. In the context of QI, the relation might hence give us a model which locates the indeterminacy in a particular observable, e.g. an electron's spin along a given axis, and it furthermore delineates all the possible values it could take, e.g. $\operatorname{spin}_{\mathrm{UP}}$ and $\operatorname{spin}_{\mathrm{DOWN}}$ on the given axis.

Before we dive deeper into the details of the theory, let us pause to provide a more intuitive illustration of what the glutty version of PMS does. Its core idea is that in a case of metaphysical indeterminacy regarding $p$, a glut of ersatz possible worlds which diverge regarding whether $p$ is or is not true manage to precisely characterize the indeterminate state of the world regarding $p$. How can that work? Taking a step back, we can see that the key to answering this question is to provide an explanation of how a range of conflicting alternatives bundled together manage to paint a complete and precise picture of a complex situation involving indeterminacy regarding a particular aspect of the situation. Taking this observation as its starting point, our illustration takes the form of an analogy. ${ }^{14}$

\footnotetext{
${ }^{11}$ The notion of possibility here and in the remainder of the paragraph must be wider than physical/nomic possibility in the sense that it allows for completely precise ersatz worlds to count as possible precisifications of reality. Logical possibility fits this bill, but so might metaphysical possibility.

${ }^{12}$ Note that "state of affairs" is here used for illustrative purposes only and is not meant to indicate that our theory presupposes an ontology of states of affairs.

${ }^{13}$ As we will see, in the case of the QI, none of those logically possible states of affairs can nomologically obtain.

${ }^{14}$ We are grateful to an anonymous reviewer for helping us clarify the analogy and the following explanation.
} 
Imagine a population of ice cream lovers, a group of people who not only have a general taste for ice cream, but also share a curious trait: for each ice cream flavour available to them, any member of the group has a determinate disposition to either like, or not like it. If you would offer them a sample of any one of these flavours and ask them whether they liked it, all members of the group would be able to immediately answer either with a clear 'yes' or 'no'. As it turns out, the preferences of the group are surprisingly homogeneous. Regarding the flavours available to them, all members of the group agree on whether they like it or dislike it. They do so, because for every such flavour they share the same disposition which either compels them to like, or to not like it. Now imagine that an artisanal ice cream producer develops a new ice cream flavour called "mangrue". A peculiar thing about mangrue ice cream is that our ice cream lovers do not have the same kind of disposition for mangrue flavoured ice cream which they have for all the other flavours. Tasting mangrue ice cream leaves them puzzled and unable to say whether they like it or not.

Now imagine that you are tasked by the Society for the Appreciation of Group Preferences ${ }^{\mathrm{TM}}$ to find out which ice cream flavours the group as a whole likes and dislikes. Being new to the job, the only thing you know is that ice cream is immensely popular in this particular group and that you have a tight deadline to submit the results. Along with a lot of ice cream samples, you are equipped with a data entry terminal and a rather basic polling software. For each ice cream lover, the software records a data set associating each ice cream flavour with a single binary value whose two possible values stand for "like" and "dislike" respectively. The software expects a complete data-set for each person, so in order to enter the data at all, the participants have to answer with either "like" or "dislike" regarding any flavour. There is no option for anyone to either refrain from answering, or to enter two values for an item.

Having no disposition to either like or dislike mangrue, the participants in the study ask you what to do if they neither definitely like, nor definitely dislike a flavour. Knowing the software and its rather crude limitations, you tell them to just randomly enter "like" or "dislike" and reassure them that you will make a note of this particular item and take care of the garbage data when analyzing the results. After completing the tasting and polling stage of the study, you proceed to inspect your collected data and notice the, to you, rather surprising agreement regarding all the other ice cream flavours, as 
well as the random fluctuation between "like" and "dislike" regarding mangrue among members of the group. After a minute of disgruntled silence, it dawns on you: What you thought to be garbage data actually provides you with an accurate model of the group's preferences!

The data sets for each individual participant are complete and contain a determinate value for every ice cream flavour. Each one considered on its own hence misrepresents the group's preference, since it gives one determinate answer, namely about whether mangrue is liked, where no such answer should be given. But this does not mean that these data sets cannot be used to accurately represent the group's preference. It turns out that you just need to carefully distinguish between complete and partial, correct and incorrect, as well als the individual's and the group's representations of preferred ice cream flavours. Since the individual data sets all agree regarding the value for all flavours except mangrue, each partially manges to correctly represent the group's overall likes and dislikes. With respect to these flavours, the group's likes and dislikes are simply identical to those of the individuals. But each individual data set also fails to correctly represent the group's complete preference, since it contains a value for mangrue which does not reflect the group's preference or distaste. But what is the group's preference or distaste regarding mangrue? It seems the right thing to say is that it is indeterminate. It turns out that there is a way of correctly representing this aspect of the the group's preference by relying only on the mostly, i.e. partially correct, but partially incorrect individual data sets: the fact that the group as a whole does not agree on whether they like or dislike mangrue already tells us that the group's complete preference-profile for ice cream flavours is indeterminate with respect to one of its aspects, namely the group's like or dislike of mangrue flavoured ice cream. This means that you can have a representation of the preferences of the group that is both complete and correct. The only unusual thing is that your indicator for indeterminacy is not a lack of recorded values, but rather a surplus. Since the ice cream lovers are a large group and the set-up of the poll allowed no coordination between them, all the values are present. But as we have seen, we can just interpret this glut of values as pointing us to an indeterminate aspect of the small piece of reality which you were supposed to capture in your experiment. Your job is done. You can finally call it a day and enjoy a fine scoop of mangrue ice cream! 
In light of the analogy let us return to PMS to see how the theory models MI and QI. Importantly, the theory does not provide a reductive definition, but rather provides a framework which allows us to model indeterminacy. Assume that our universe is indeterminate with respect to whether $p$ is the case, but otherwise determinate. PMS allows us to construct an intended model of this scenario by first sorting the totality of the ersatz worlds into those which incorrectly represent all determinate aspects of reality (i.e. everything except what's captured by $p$ ) and those which correctly represent them and only admitting the worlds in the latter category into our model. This is the job of the plural precisification-relation $R_{p p}$. Like the data sets constrained by the limitation of the polling software in our analogy, each ersatz world in the model individually gives us a definite answer concerning whether any proposition about our universe is true or false and therefore individually give us a complete, but on the whole incorrect representation of our universe, since they misrepresent it with respect to $p$ (and only with respect to $p$ !). This is not a problem, since in PMS, no single ersatz world has to represent reality as a whole correctly. This is simply not the theoretical role it has to play. Rather, the fact that some worlds in the intended model represent $p$ as true and some as false, gives us a correct representation of the way in which our universe is indeterminate, since the plurality of all worlds in the model gives us the correct truth values for all propositions which capture determinate aspects of reality (all ersatz worlds agree on them) and a truth value glut of both true and false regarding $p$ (the ersatz worlds disagree in the truth value they assign), the proposition which is about the indeterminate aspect of reality. From an intuitive perspective, the core idea of the glutty version of PMS is hence that, just as the indecision of a group as a whole is sometimes best understood in terms of definite individual opinions which agree on most, but diverge on certain items, cases of metaphysical indeterminacy are sometimes best understood in terms of a coincidence of several ersatz worlds which agree on the truth or falsity of most, but disagree on the truth or falsity of some propositions, namely those which are about the indeterminate aspects of reality.

A diverse class of potential examples of MI have been given in the literature. Some for example claim that the openness of the future should be understood in terms of MI (e.g. Barnes \& Cameron 2008) others link MI to material objects with fuzzy boundaries, (e.g. Tye 1990) and there are of course those who argue that quantum mechanics give rise to MI (see e.g. 
Darby 2010, Skow 2010, Calosi \& Wilson 2019). It is not clear whether these examples all involve the same sort of MI. We believe that Plural Metaphysical Supervaluationism (PMS) is flexible enough to accommodate these different sorts of MI, as long as they can in principle be treated by supervaliationist means.

A core question about any version of supervaluationism is how it handles the notion of truth. The standard approach in semantic supervaluationism is to identify truth with super-truth, (see Keefe 2000 , §8) i.e. truth in all admissible precisifications, but the framework of PMS offers the resources to define different notions of truth. In the application of the theory to cases of QI, we will rely on the notion of super-truth to give us a notion which tracks what the quantum state tells us about the states of the world. In such cases, there will as a consequence be certain propositions which fail to express super-truths. Accordingly, we will, in one sense, not have a classical, bivalent object language. We can however make use of the flexibility of the framework to define classical notions of logical truth and consequence. This bi-furcated approach to truth will be introduced and motivated in the following subsections.

Our treatment of logical truth differs importantly from that proposed in Barnes \& Williams 2011. B\&W define logical truth and consequence in terms of truth in a model and truth in a model in terms of truth in the designated world of a model (cf. p. 133 for the definition in the final third version of their theory). This definition fits the core idea of their theory, namely that metaphysical indeterminacy is indefiniteness in which one of a range of candidate ersatz worlds is actualized. Since PMS allows cases in which no single world in a model is on its own a candidate for being the one actualized world, a definition of logical truth relying on designated worlds would not be the best fit. The definition of logical truth we adopt in the next subsection does not rely on designated worlds and is hence a better match for PMS.

\subsection{Semantics and Logic}

\subsubsection{Model Theory and Semantics}

In the previous subsection, we have presented the main idea of plural metaphysical supervaluationism by focusing on the relation $R_{p p}$, the relation which holds between reality and the plurality of ersatz worlds used to model QI in our theory. While this relation is an important component of PMS, it 
does not belong to the supervaluationist semantics proper which provides the formal component of the core of the theory. With respect to the latter, our approach is to stay as conservative as possible to semantic supervaluationism. What then is the connection between $R_{p p}$ and the semantics? To answer the question let us introduce the version of supervaluationist semantics we work with.

The semantics is a semantics for the language of propositional logic with the standard set of logical connectives enriched with the $D$-operator which syntactically acts just like negation, forming a formula when prefixed to a formula and which allows us to express claims about determinacy and indeterminacy. We will use negation and conjunction as the basic logical connectives. Well-formedness of logically complex formulas is defined in the standard way. We are focusing on a propositional language instead of a first-order language (unlike Barnes \& Williams 2011) in presenting the semantics and logic since this somewhat simplifies the presentation and this fully suffices to spell out the two crucial differences between our theory and standard semantic supervaluationism. Just like in B\&W's semantic, the $D$-operator expresses a primitive concept of definiteness and can be used to syntactically define the notion of indeterminacy ("It is indeterminate whether...") in the standard way: Where $\varphi$ is a formula of our formal language, that it is indeterminate whether $\varphi$ is expressed by the formula $\neg D \varphi \wedge \neg D \neg \varphi$ ("It is not definite that $\varphi$ and not definite that not- $\varphi$ "). ${ }^{1516}$

The semantics is a standard possible-worlds semantics for the language of propositional logic enhanced with the $D$-operator. A model $M$ is an ordered triple $<W^{M}, R^{M}, \llbracket \cdot \rrbracket^{M}>$, where $W^{M}$ is a non-empty set of (ersatz) possible worlds, $R^{M}$ a binary accessibility relation which relates pairs of worlds taken from $W^{M}$, and $\llbracket \cdot \rrbracket^{M}$ a valuation function which assigns one of the two truth-values TRUE and FALSE to each formula relative to each possible world in $W^{M}{ }^{17}$

\footnotetext{
${ }^{15}$ We rely on the context to distinguish between use and mention throughout this section and the whole paper.

${ }^{16}$ Note that this definition does not amount to a reductive analysis of indeterminacy; like B\&W's theory, our theory is non-reductive, as indicated in section 3.1.

${ }^{17}$ Note that this means that our semantics corresponds to a restricted variant of Fine's supertruth-based semantics developed in Fine (1975) which is limited to complete models and in which models correspond to possible worlds. It closely follows the semantics for semantic supervaluationism due to Asher et al (2009).
} 
The core idea of the semantics is to treat the $D$-operator as a necessity-operator. Semantically, this means that it is interpreted in terms of universal quantification over accessible worlds. With $p$ an arbitrary propositional constant, $\varphi, \psi$ formulas of our formal language, and using $M, w^{\Vdash} \varphi$ to express that formula $\varphi$ is true in world $w$ in model $M$, we can then recursively define truth in a world in a model as follows:

\begin{tabular}{|c|c|c|}
\hline $\begin{array}{ll}\text { - } & M, w \Vdash p \\
\text { - } & M, w \Vdash \sim \varphi \\
\text { - } & M, w \Vdash \varphi \wedge \psi \\
\text { - } & M, w \Vdash D \varphi\end{array}$ & $\begin{array}{l}\text { iff } \\
\text { iff } \\
\text { iff } \\
\text { iff }\end{array}$ & $\begin{array}{l}\llbracket p \rrbracket^{M, w}=\text { TRUE. } \\
M, w^{\nVdash} \varphi \\
M, w^{\Vdash} \varphi \text { and } M, w^{\Vdash} \psi \\
\text { for all } w^{\prime} \text { such that } w R^{\mathrm{M}} w^{\prime}, M, w^{\prime} \Vdash \varphi\end{array}$ \\
\hline
\end{tabular}

The other logical connectives are defined in terms of negation and conjunction in the usual way.

So far, the semantics is not just conservative with respect to (a possible worlds-based version of) supervaluationist semantics, it is in fact identical to it. What distinguishes our semantics from that of semantic supervaluationism are two features which are not visible in the core semantics provided by the definition of truth in a world in a model.

The first distinctive feature of our theory is that the relation $R_{p p}$ (which is not to be confused with the model-relative accessibility relation $R^{\mathrm{M}}$ at work in the semantics proper!) puts a constraint on the models our theory operates on. Which constraint is that? We have previously introduced this relation as a relation which holds between the actual universe and a plurality of ersatz worlds, where the former is the concrete entity containing us and everything else with which we are in direct or indirect causal contact and the latter are abstract objects used in philosophical theories. So construed, $R_{p p}$ always has the same entity, the actual universe, as its first relatum, but may relate it to distinct sets of ersatz worlds, allowing us to model MI in the actual world. In practice however, we want the relation to do the same for non-actual states of the universe, or more generally for non-actual possible worlds, since e.g. we may want to reason about counterfactual cases of MI, or since we simply do not know enough about the state of our universe to select a unique set of ersatz worlds to capture its indeterminacy.

Generalizing to non-actual universes, $R_{p p}$ relates an actual or possible universe to a plurality of ersatz worlds which, assuming the second interpretation of the relation proposed in the previous section, together give 
us a precise picture of that universe and including both its determinate, and indeterminate aspects. For any model $M, R_{p p}$ thereby determines whether that model accurately represents the state of any universe, including its determinate (and in the relevant cases) also its indeterminate aspects by relating that universe to the plurality of worlds which form the set $W^{M}$ of ersatz worlds of $M$. Admissibility in PMS is hence a relative matter: models are admissible relative to the actual or a possible universe, not admissible tout court.

Let us briefly pause to comment on the relation between $R_{p p}$ and $R^{M}$. We have earlier introduced $R_{p p}$ as the plural precifification relation, but we can now see that this description, while still capturing the general role the relation plays in our theory, is somewhat misleading. In supervaluationist semantics, the label "precisification relation" is usually applied to the accessibility-relation which holds between worlds in a model. In our model theory, this accessibility-relation is $R^{M}$ and not $R_{p p}$. It is hence more apt to call $R_{p p}$ the admissibility relation, since it determines which models are admissible as models of the (indeterminate) state of reality or of a merely possible universe. $R_{p p}$ does this by picking out a plurality of ersatz worlds (the elements of $W^{M}$ ) which then in turn stand in the relation $R^{M}$ in any admissible model $M$.

One might wonder about the metaphysical status of the possible universes which $R_{p p}$ relates to pluralities of ersatz worlds. Are they concrete universes in the sense of Lewis's modal realism, ersatz worlds, like those at work in the formal part of our theory, or something else? Given our reliance on ersatz worlds, the modal realist option is unattractive for reasons of parsimony. The disadvantage of relying on ersatz worlds is that it entails that $R_{p p}$ no longer neatly bridges the gap between the, metaphysically speaking, actual world and the possibly actualized ersatz worlds which together model its indeterminacy in PMS. One further proposal which allows us to sidestep thorny metaphysical issues regarding the nature of possible worlds is to take a page out of the two-dimensionalist's book ${ }^{18}$ and to assume that instead of the counterfactually possible ersatz worlds at work in our models, $R_{p p}$ takes an counteractually possible world as its first relatum, where the distinction between counterfactual and -actual is not metaphysically substantive, but rather a matter of perspective or theoretical role. We will not take a definitive stand regarding this issue here. In order to

\footnotetext{
${ }^{18}$ Cf. for example Chalmers 2006, especially $§ 3$.
} 
develop the formal part of our theory, the only thing which we need to assume is that we are not limited to our actual universe as the only first relatum of $R_{p p}$.

The second distinctive feature of our logic and semantics is that due to its function as a $W^{M}$-determiner, $R_{p p}$ (in its generalized form) can naturally be taken to put a constraint on the interpretation of the accessibility relation $R^{\mathrm{M}}$ of any possibly admissible model $M$ and consequently on the logic of the $D$-operator with respect to this class of models. We will come back to this point in $\$ 4.2 .3$, but let us now focus on the definitions of logical truth and consequence.

\subsubsection{Logical Truth and Consequence}

Our semantics is designed to admit cases of indeterminacy, i.e. cases in which a formula is neither definitely true, nor definitely not true. According to the standard version of semantic supervaluationism, truth simpliciter, i.e. the notion of truth which connects the theoretical notion of truth in a world in a model back to the ordinary notion of truth, is identified with supertruth. (Keefe 2000, §8). In our models, this identification would give rise to truth-value gaps, assuming that supertruth in a model is defined as truth in all worlds of the model, since a formula which is neither definitely true, nor definitely false with respect to one world in the model will be neither supertrue, nor superfalse. Does this mean that our theory gives us a non-classical logic? The definition of logical consequence and truth presented in this subsection allows us to stay classical, meeting a desideratum expressed both in Barnes \& Williams (2011) and Calosi \& Wilson (2019), which could be contested, but which we accept for the sake of the argument.

One of the main objections to semantic supervaluationism is that as soon as a definitely-operator is introduced into the object language of the theory, it stops being fully classical. More specifically, Williamson has argued that the theory invalidates some inference patterns which are valid in classical logic, including in particular contraposition, proof by cases, and indirect proof (see Williamson 1994, §5.3). The failure of these patterns can be traced to the fact that standard supervaluationism, which relies on supertruth to define the notion of logical consequence, invalidates the deduction theorem. ${ }^{19}$

${ }^{19}$ I.e. where $\varphi, \psi$ are formulas, $\Delta$ is a set of formulas, and $\vDash_{S}$ is the supertruth-based 
There are different ways around this problem for supervaluationism (including both its semantical and metaphysical variants) which we can rely on to avoid deviations from classical logic in the context of PMS. In $§ 3.1$ we have already pointed out that $B \& W$ rely on a definition of logical consequence based on the designated actualized worlds of models. Since our models do not contain a designated actualized world, we have to take a different approach. We adopt the one proposed by Asher et al (2009) which is based on two assumptions. The first is that supervaluationism provides us with the means to define different notions (or modes) of truth which may serve different theoretical purposes (Asher et al, 2009, p. 916). This in particular allows us to distinguish the notion of truth used in the definition of logical truth and consequence from the notion of supertruth in a model, which we rely on in order to account for QI.

The second idea is that the mentioned deviations from classical logic can be avoided by relying on a particular notion of logical consequence. As Williamson points out in his critique of supervaluationism and as Asher et al (2009) elaborate, supervaluationists who want to stay classical logic-wise can replace the global notion of logical consequence they standardly rely on with a local notion. Let $\varphi$ and $\Gamma$ respectively denote a formula and a set of formulas of our formal language. We can then define a local notion of supervaluationist consequence symbolized by $\vDash$ as follows (Asher et al, 2009, p. 919):

- $\quad \Gamma=\varphi \quad$ iff $\quad$ for every model $M$ and every world $w$ in $W^{M}$, if $M, w \Vdash \Gamma$, then $M, w \Vdash \varphi$.

Logical truth is defined in terms of logical consequence from the empty set of formulas in the standard way:

$$
\text { - } \vDash \varphi \quad \text { iff } \quad \varnothing \models \varphi \text {. }
$$

notion of logical consequence, it is not generally the case that if $\Delta \cup\{\varphi\} \vDash_{s} \psi$, then $\Delta \models_{S} \varphi \rightarrow \psi$. See Fine 1975, p. 290 and Asher et al 2009, p.909. Counterexample: let $\Delta$ be empty and substitute $p$ for $\varphi$ and $D p$ for $\psi$. With $\vDash_{\mathrm{S}}$ expressing conservation of supertruth, i.e. conservation of truth in all worlds in all supervaluationist models, we have that if $\vDash_{\mathrm{S}} p$, then $\vDash_{\mathrm{S}} D p$, but not that $\vDash_{\mathrm{S}} p \rightarrow D p$, since there are models containing worlds in which $p$ is true, but not $D p$. 
Since each world gives us a classical valuation of our language, the definitions ensure that the notions of logical consequence and truth coincide with their classical counterparts for all formulas which do not contain the $D$-operator. The deviations from classical logic involving the operator are also avoided: they result, as mentioned earlier, from the failure of the deduction theorem in standard supervaluationist logic, but the deduction theorem holds for $\vDash .{ }^{20}$ The cost of conserving classical logic in this manner is that the inferential logic for the $D$-operator fails to validate the rule of introduction for the $D$-operator (D-intro), the rule which allows us to infer from $\Delta \models \varphi$ to $\Delta \models D \varphi$, where $\Delta$ is a set of formulas. ${ }^{21}$

Note that the quantifier in the definition of logical consequence is unrestricted, notably ranging over all logically possible models and in particular over models which are inadmissible in the context of an application of PMS to a case of QI, or a case of MI more generally. This should not come as a surprise, since whether a model is admissible as a model for a particular case of MI should not have an impact on the meaning of the notions of logical truth and consequence. To put it differently, logic makes no difference between models which serve that particular philosophical purpose and those which do not. The permissive approach regarding the diverse notions of truth definable in supervaluationism naturally allows us to disentangle the notion of logical truth and that of determinate truth in a model which is admissible in a case of MI or QI in the manner just suggested.

\subsubsection{The logic of the D-operator}

Let us come back to the second distinctive feature of the formal part of our theory, the $R_{p p}$-based interpretation of the accessibility relation $R^{M}$ and of the logic of the $D$-operator for the subclass of all possibly admissible models. $R_{p p}$ selects the worlds contained in the set $W^{M}$ of any such model $M . R_{p p}$ 's role as the plural precisification relation strongly suggests an intuitive interpretation of the accessibility-relation $R^{M}$ within each such model $M$,

\footnotetext{
${ }^{20}$ I.e. we have that if $\Delta \cup\{\varphi\} \vDash \psi$, then $\Delta \vDash \varphi \rightarrow \psi$. The counterexample described in note 16 does not arise, since $p \models D p$ requires the conditional $p \rightarrow D p$ to be true in all worlds in all models, which just means that $\vDash p \rightarrow D p$.

${ }^{21}$ See Asher et al (2009), p. 922-3. This feature of the logic is incidentally very much in line with the way cases of QI are handled in PMS, since in these cases, the determinate truths are imposed on the intended model by the physics. See $§ 5.3$.
} 
which is directly relevant to its formal properties and hence also to the partial logic of the $D$-operator with respect to the set of all possibly admissible models. The worlds in $W^{M}$ are the worlds which together, assuming again the second proposed interpretation of $R_{p p}$, give us a precise picture of the possible world modelled by $M$, including its indeterminate aspects. Accordingly, $R^{M}$ can naturally be taken to be the relation of co-precisification, the relation which holds between two worlds $w$ and $w^{\prime}$ just in case $w$ contributes to precisifying an actual or merely possible universe together with $w^{\prime}$ in $M$.

Note that this interpretation of $R^{M}$ as the relation of co-precisification takes into account both non-reducible and reducible cases of plural precisification. The difference between the two sorts of cases is that in the former, $w^{\prime}$ has to substantially contribute to precisifying our universe, whereas in the latter, the contribution of $w^{\prime}$ might be insubstantial, since $w$ alone (as well as $w^{\prime}$ alone) might already qualify as a precisification.

What is the result of interpreting the accessibility relations in possibly admissible models as relations of co-precisification among plural precisificational possibilities? First of all, this interpretation renders the logic of the $D$-operator's a normal modal logic, as suggested by Asher et al (2009). This means that it contains the axioms of propositional logic and that we have the rules modus ponens, uniform substitution, and the rule of necessitation:

- Rule of necessitation: If $\vdash \varphi$ then $\vdash D \varphi$

Furthermore, we have the K-axiom:

- K-axiom: $\quad D(\varphi \rightarrow \psi) \rightarrow(D \varphi \rightarrow D \psi)$

The rule of necessitation and the $\mathrm{K}$-axiom for $D$ place rather minimal constraints on the logical behaviour of the operator which are completely in line with interpretation in terms of the notion of co-precisification it is given in PMS. It is furthermore easy to justify the inclusion of the T-axiom, which holds in all models in which $R^{M}$ is reflexive:

- T-axiom: $\quad D \varphi \rightarrow \varphi$ 
Given our interpretation, $R^{M}$ is reflexive since any world selected by $R_{p p}$ contributes (substantially or insubstantially) to precisifying the actual universe in a possibly admissible model $M$ together with itself. The resulting logic KT is the weakest plausible logic for the $D$-operator. As we have just seen, the interpretation of the accessibility-relation as the relation of co-precisification guarantees that the partial logic over the set of possibly admissible models is a logic of this kind.

The logic of the $D$-operator should minimally be as strong as KT, but is it stronger? Like the third and final formal system presented in Barnes \& Williams (2011), our interpretation of $R^{M}$ entails that it is S5: The relation of co-precisification is an equivalence relation, i.e. in addition to being reflexive, it is also both symmetric (if $w$ is a co-precisification of $w^{\prime}$, then $w^{\prime}$ is a co-precisification of $w$ ) and transitive (if $w$ is a co-precisification of $w$ ' and $w^{\prime}$ is a co-precisification of $w^{\prime \prime}$, then $w$ is a co-precisification of $\left.w^{\prime \prime}\right)$, which means that the partial logic of $D$ contains the B- and 4-axioms respectively, giving us $\mathrm{S} 5$ :

- B-axiom $\varphi \rightarrow D \sim D \sim \varphi$

- 4-axiom $D \varphi \rightarrow D D \varphi$

Note that the same arguably holds for the weaker first interpretation of $R_{p p}$, since the relation of failing-to-determinately-misrepresent-reality-together-with is plausibly also an equivalence relation.

To summarize, PMS $\operatorname{can}^{22}$ incorporate a supervaluationist logic for a formal language containing the $D$-operator which is classical and gives us the analogue to a normal modal logic as strong as S5 with respect to the class of all possibly admissible models. This all but guarantees that the same holds for the full logic, i.e. the logic as defined in $\S 4.2 .2$ with respect to both possibly admissible and necessarily inadmissible models: no possibly admissible PMS model is a countermodel to S5, so the complete logic could only turn out to be weaker for general reasons wholly independent of PMS.

\footnotetext{
${ }^{22}$ But need not. As we have mentioned in $\S 4.2 .2$, the semantics we present here meets the classicality desideratum formulated by Barnes and Williams and Calosi and Wilson. If one rejects this desideratum, one can instead develop a version of PMS which incorporates a non-classical logic and semantics.
} 
A particular feature of the version of PMS developed in this section is that its notion of logical truth is not defined in terms of supertruth, i.e. truth in all worlds in a model. Yet, the latter nation still has an important role to play in our theory, as we will see in the next section.

\section{$5 \quad$ Modeling Quantum Indeterminacy with PMS}

In this section our aim is to show how PMS is to be applied to a concrete case, namely quantum indeterminacy (QI). In $\$ 5.1$ we introduce three objections raised against standard MS that are based on quantum mechanical phenomena. In $\$ 5.2$ we discuss the proposals recently made by Torza (2019) and Darby \& Pickup (2019) to escape one of these objections, and suggest why one might find them unsatisfactory. In $\S 5.3$ we show how PMS addresses the objections.

\subsection{The QM Objections to MS}

Skow (2010) and Darby (2010) independently argued that MS cannot account for indeterminacy in QM. To see why, let us start by asking how MS would apply to QI. A natural thought is to interpret each 'side' of a superposition state as a precisificational possibility. Suppose we perform a spin measurement on a quantum system $S$ in the direction $\mathrm{z}$, and find the system in an eigenstate of $\operatorname{spin}_{\mathrm{UP}}$ :

$$
S=\mid \uparrow z>
$$

Different spin components in QM are incompatible observables, which means that if a system $S$ is in eigenstate of spin along a given axis $(z$, in our example), then $S$ is in a superposition of the directions mutually orthogonal with $z$ (namely, $x$ and $y$ ):

$$
\begin{aligned}
& S=|\uparrow z>=1 / \sqrt{ } 2| \downarrow x>+1 / \sqrt{ } 2 \mid \uparrow x> \\
& S=|\uparrow z>=1 / \sqrt{ } 2| \downarrow y>+1 / \sqrt{ } 2 \mid \uparrow y>
\end{aligned}
$$

According to MS, the following are true in a model representing the above states:

1. $D\left(S_{\text {z-spin }}=\uparrow\right)$

2. $D \neg\left(S_{\text {z-spin }}=\downarrow\right)$ 


$$
\begin{array}{ll}
\text { 3. } & \neg D\left(S_{\text {x-spin }}=\uparrow\right) \& \neg D \neg\left(S_{\text {x-spin }}=\uparrow\right) \\
\text { 4. } & \neg D\left(S_{\text {x-spin }}=\downarrow\right) \& \neg D \neg\left(S_{\text {x-spin }}=\downarrow\right) \\
\text { 5. } & \neg D\left(S_{\text {y-spin }}=\uparrow\right) \& \neg D \neg\left(S_{\text {y-spin }}=\uparrow\right) \\
\text { 6. } & \neg D\left(S_{\text {y-spin }}=\downarrow\right) \& \neg D \neg\left(S_{\text {y-spin }}=\downarrow\right)
\end{array}
$$

In words, $S$ is definitely $\operatorname{spin}_{\mathrm{UP}}$ and definitely not $\operatorname{spin}_{\mathrm{DOWN}}$ along $z$, and it is indeterminate whether $S$ is $\operatorname{spin}_{\mathrm{UP}}$ along $x, \operatorname{spin}_{\mathrm{DOWN}}$ along $x$, $\operatorname{spin}_{\mathrm{UP}}$ along $y$, and $\operatorname{spin}_{\mathrm{DOwN}}$ along $y$. (3)-(6) are spelled out in terms of the (standard MS) $D$-operator in the usual way (cf. §4.2.1). Semantically, (1)-(2) tell us that, according to every ersatz world in the MS model representing the state of $S$, $S$ has $\operatorname{spin}_{\mathrm{UP}}$ in $z$. (3)-(6) express that this is not the case for the spin states along $x$ and $y$. There is no uniform value (up or down) which these observables have across all the ersatz worlds in the model. So far, so good.

A serious problem arises once we look more closely at each ersatz world in the model and think about whether it is indeed a precisification in the sense of standard MS, i.e. an ersatz world which does not determinately misrepresent the system. To answer this question, we have to ask which values $x$-spin and $y$-spin $S$ have in the ersatz worlds of the model. Given that the state of the system demands that each precisification has to agree on the value of $z$-spin (otherwise, they would definitely misrepresent reality), the following conjunctions represent the only four ersatz worlds in the model which qualify as candidates for being admissible precisifications (leaving out the negations of the opposite spin states for the sake of simplicity):

$$
\begin{aligned}
& w 1:\left(S_{\mathrm{z} \text {-spin }}=\uparrow\right) \&\left(S_{\mathrm{y}-\mathrm{spin}}=\downarrow\right) \&\left(S_{\mathrm{x} \text {-spin }}=\downarrow\right) \\
& w 2:\left(S_{\mathrm{z} \text {-spin }}=\uparrow\right) \&\left(S_{\mathrm{y}-\mathrm{spin}}=\uparrow\right) \&\left(S_{\mathrm{x} \text {-spin }}=\downarrow\right) \\
& w 3:\left(S_{\mathrm{z} \text {-spin }}=\uparrow\right) \&\left(S_{\mathrm{y} \text {-spin }}=\downarrow\right) \&\left(S_{\mathrm{x} \text {-spin }}=\uparrow\right) \\
& w 4:\left(S_{\mathrm{z} \text {-spin }}=\uparrow\right) \&\left(S_{\mathrm{y} \text {-spin }}=\uparrow\right) \&\left(S_{\mathrm{x} \text {-spin }}=\uparrow\right)
\end{aligned}
$$

The reason why every $(w 1)-(w 4)$ has to specify a value for each spin property is because, recall, according to MS ersatz worlds, that is presifications, are maximally complete sets of propositions. Thus, they cannot leave matters unsettled. This is however a highly problematic assumption when it comes to QM. In effect, if we were to write down the states of the system $S$ for each of (wl)-(w4), we would end up having states that are nomologically impossible, since they are ruled out by QM. If we consider $w 1$, for instance, the state of $S$ would be something like: 


$$
S=|\uparrow z>+S=| \downarrow y>+S=\mid \downarrow x>
$$

This state however makes no sense in QM, because assignments of spin values in three mutually orthogonal directions have to satisfy the following constraint in order to be consistently represented in the Hilbert space: $\mathrm{n} S x+$ $n S y+n S z=2$. In other words, one of the three directions has to be assigned value 0 , which basically entails that either of the three conjuncts in $w 1$ cannot be true.

Observables (like spin) in QM are represented by operators in a multidimensional vector space. When an operator gets value 1 (so: the vector with which it is associated is an eigenvector), its corresponding subspace has value 1. Crucially however, different operators (corresponding to different observables, such as different spin components) may share the same subspace (and so the same value associated with it). This is roughly why assignments of values must satisfy the constraints inherited from the vectorial space, and in the case of mutually orthogonal spin components they cannot always be assigned a value.

Darby and Skow exploit this mathematical feature of the Hilbert space formalism to show that the core tenet of MS according to which precisifications are precise is in tension with quantum mechanics. We register that they also go a step further when they claim that the Kochen-Specker theorem (KS, 1967) is essential to get to this result. However, it is not clear why this should be the case, so let us say a few words to clarify this. Theorems such as the KS theorem exploit the relations between specific value assignments in subspaces for Hilbert spaces of certain dimensions and a specific number of vectors ${ }^{23}$ in order to generate concrete cases where observables cannot be assigned a value (unless we assign two incompatible ones, 0 and 1). These theorems are meant to rule out a certain class of theories (those which posit the so-called hidden variables) which regard the impossibility of assigning every value as an epistemic limitation. In this sense, the theorems play quite an important role

${ }^{23} \mathrm{KS}$ establishes a contradiction for a Hilbert space of 3 dimensions with 117 vectors, and this is why the proof is particularly complicated. A simpler proof is in Cabello at el (1996), where the Hilbert space is $4 d$, with 18 vectors. Cabello's proof is however weaker, because any contradiction in $3 d$ is also a contradiction in $4 d$ while the converse is not true. 
in quantum foundations. However, it seems misleading to refer to them in the context of a debate over whether MS is compatible with quantum indeterminacy or not. After all, if we were to regard the impossibility to assign definite values as epistemic, then why bother applying MS in the first place? If the indeterminacy would just be epistemic, ${ }^{24} \mathrm{MS}$ would trivially not apply anyway.

To recap: (i) in MS precisifications are fully precise sets of propositions; (ii) in QM it is impossible to always assign precise values to certain mutually dependent observables; (conclusion) MS cannot account for quantum indeterminacy. Clearly, it is (i) that defenders of standard MS will want to reject by revising the account. As we are about to show, this can be done in various ways. Before that, however, we have to present two further objections to MS based on quantum mechanics, both raised by Calosi \& Wilson (2019).

Calosi \& Wilson argue that the incompatibility between quantum indeterminacy and MS goes much deeper than what Skow and Darby realised, and concerns the very fact that the account attempts to understand indeterminacy as a meta-level phenomenon, as Wilson calls it. They consider two important features of quantum mechanics, and argue that MS is utterly unable to account for them. First, they consider the phenomenon of quantum interference (as in the double-slit experiment), and ask what explains the presence of the interference patterns according to MS. After all, we see interference effects as due to superposition. This suggests that a superposed state has physical effects. The question then is how can we understand the effects of superposition states if these are understood as the world being unsettled as to which precisification is actualised? Can it be that presicifications, which are by definition abstract entities, interact with each other to form the typical interference pattern? At the very least, such a view would entail a substantive departure from the spirit of MS.

Secondly, Calosi \& Wilson suggests that MS fails to provide a plausible explanation for quantum statistics. This is indeed quite straightforward. If we take an equal-weight superposition, say of an electron being $50 \%$ up and

${ }^{24}$ Some interpretations of QM maintain that this is the case at the price of considering observables like spin as contextual (i.e. dependent on the measurement context). Since non-contextuality is an assumption of KS, by dropping we avoid value indefiniteness. That is why, to be precise, what the theorems establish is the impossibility of non-contextual hidden-variables theories. 
$50 \%$ down, perhaps the claim that the world is unsettled between two options might look intuitive. Consider however a state like the following:

$$
\mathrm{S}=75 \%|\downarrow y>+25 \%| \uparrow y>
$$

How is the machinery of MS supposed to account for this case? It seems fair to say that having just two presicifications, without further ado, simply will not do.

We can thus distinguish three objections to MS based on quantum mechanics. The first, identified by Skow and Darby, is what we dub the Indefinite-Value Objection. The second and third, identified by Calosi \& Wilson, will be called the Interference-Pattern Objection and the Statistics Objection. In $\$ 5.2$ we focus on two responses to the Indefinite-Value Objection to be found in the recent literature. We will then come back to our own proposal, and to how it responds to each objection, in $\S 5.3$.

\subsection{Sophisticated Versions of MS}

In the recent literature on MI there are two proposals that are somewhat similar to ours, in the sense that both are in part an attempt to "save" the spirit of the B\&W account from the objection that it cannot model the quantum case. The focus of these proposals is squarely on the Indefinite-Value Objection. As we have seen, the core of this objection is that for systems that show a quantum behavior, it is impossible to be in a determinate state for each value of their incompatible observable properties. If metaphysical indeterminacy is indeterminacy with respect to which of many globally determinate states of the system is the actual one, then it is determinately false that any of them is. In order to save the meta-level approach from this glitch, an all but obvious idea is to change the semantic machinery from one in which evaluation is carried out at a global level, to one in which the evaluation concerns only local matters. There are two ways, at least, to implement the idea. One is Darby and Pickup's modification of the point of evaluation-from whole possible worlds to situations $^{25}$ - the other is Torza's modification of the semantic relation of representational adequacy involved - from a bivalent one (because based on complete representations) to one which is non-bivalent (because based on

\footnotetext{
${ }^{25}$ They adopt this notion from situation semantics. See e.g. Barwise \& Perry 1983.
} 
incomplete and yet adequate representations). We argue that both proposals face problems which do not affect ours.

The main idea of Darby and Pickup's paper is that unlike possible worlds, which are global and settle the truth or falsity of every proposition evaluable at them, situations can be silent with respect to certain matters ${ }^{26}$. This feature is exploited to model the radical lack of value determinateness typical of quantum systems. If for an electron $e$, for instance, it is determinate whether its spin is up or down with respect to the $x$-axis, then $e$ 's spin status must be indeterminate with respect to the $y$-axis. Given that a situation about $e$ 's spin status along the $x$-axis can be silent with respect to $e$ 's spin status along the $y$-axis, and vice versa, we can model in a meta-level fashion the indeterminacy involving $e$ as a form of unsettledness with respect to which of four situations ( $e$ is up along the $x$-axis, $e$ is down along the $x$-axis, $e$ is up along the $y$-axis, $e$ is down along the $y$-axis) is the actual one. None of the four situations will definitely fail to misrepresent the actual world, as a situation encompassing both a determinate value for the $x$-axis and for the $y$-axis would.

This strategy to screen off the meta-level approach from the quantum objection is suspicious for at least two reasons. Firstly, it is hard to see how a precisification can be partial. The original idea behind supervaluationism is that an imprecise representation is "molded" into a set of precise ones, and the imprecision of the representation is thereby "transferred" to the unsettledness of the semantic relation between those and reality. When adopting this idea to MI, we exploit the semantic machinery to account for the behaviour of indeterminacy talk on the assumption that there is non-representational indeterminacy in reality. But situations are not supposed to correspond to ways reality is like, unless we consider only the complete ones (with respect to an underlying bivalent logic), that is unless we fall back on the possible world-based meta-level account. Ersatz precise situations are at best partial representations of reality and thus not ways in which reality could be more precise. Our proposal, by contrast, keeps the core idea of a precisification as a way reality could be if there were no indeterminacy intact, without succumbing to the problem of ruling out the value unsettledness of quantum systems.

Secondly, it is not clear what the metaphysical status of the situations that do not misrepresent the actual world is. It seems clear from the general idea

\footnotetext{
${ }^{26}$ See Corti (forthcoming) for a critical discussion of Darby \& Pickup's proposal.
} 
of "saving" the meta-level approach from the quantum objection, that situations, like words in the proposal of $B \& W$, have an ersatz, representational nature. But unlike ersatz possible worlds, situations are typically given by combinations of some but not all of the entities and properties/relations that we find in the model. Therefore, in order to understand what a situation could represent, we need to specify the overall worldview from which we abstract them, that is we need to tell a story about the nature of the entities that inhabit those situations as inhabitants of a possible world. ${ }^{27} \mathrm{~A}$ natural option is to take them as an abstraction from the actual world. This would be akin to Barwise and Perry's original idea of understanding situations as concrete states of affairs. But in the context of Darby and Pickup's proposal this is problematic. On the one hand, the theory is silent with respect to the metaphysical status of the actual world (after all it is a meta-level and not an object level account), but on the other hand it requires that the situations that are candidate for actuality are metaphysically dependent on it. The theory thus does not specify any theoretical role for the actual world, but (implicitly) posits a dependence relation between it and situations, whose metaphysical nature is explanatorily crucial for the project. In contrast, in our theory, the actual world has an important and clearly defined part to play, since it selects the ersatz worlds used to model MI in each admissible model.

Maybe this is not a fatal strike to the project, and possibly the approach can be integrated with an object-level account of the indeterminacy in the world (although in that case, it would go against the initial aim of saving B\&W's basic idea). However, our proposal is not susceptible to the same tension, since it preserves the original idea of indeterminacy with respect to which precisification correspond to the actual world as a way to model the semantic behaviour of our talk about metaphysical indeterminacy, it only changes the relation between the precisification and reality (from a singular to a plural one).

Torza's proposal is one that explicitly (i) "rejects the precisificational view of metaphysical indeterminacy" (2017: abstract), and (ii) aims at

${ }^{27}$ Those claims would need some fine honing, given that variant domain versions of possible world semantics and the possibility of alien properties, but the point we are making here does not require such a level of refinement. Capturing the difference between global possible worlds and local situations would still require the specification of what situations are abstracted from. 
providing a reductive analysis of metaphysical indeterminacy, rather than an elucidation of the semantic behavior of a primitive notion. Those two claims seem to be in tension with the idea of a "general theory of metaphysical indeterminacy in the vicinity of [B\&W's]" (p. 3). What we argue for in what follows is that insofar as Torza's theory is seen as a strategy to save the meta-level approach to metaphysical indeterminacy from the quantum objection, then it fares worse than ours.

Torza agrees with B\&W's assumption that reality is determinate if and only if there is a unique and complete ersatz world that represents it. However, in cases of MI or of QI in particular, he takes the uniqueness condition to hold, and the completeness condition to fail. Roughly, the world is indeterminate in virtue of the fact that there can be representations of it that are neither true nor false. Of course, it is not the mere failure of the completeness condition per se that can account for the fact that the theory is providing us with a model of metaphysical indeterminacy (and a reductive one at it). If we bracket all mentions whatsoever of guinea pigs, we can construct many ersatz words that are incomplete about the subject matter of guinea pigs, and can thus be said to neither capture the truth, nor the falsity of claims that involve guinea pigs. From this hardly any fact about the indeterminate status of guinea pigs, and indeed any fact about the metaphysical status of the world at large, can be correctly inferred. That is why crucial explanatory work in Torza's account is done by the notion of a semantically nondefective language.

We agree that once this notion is taken on board, a reductive account of metaphysical indeterminacy (as "representational incompleteness in a semantically nondefective language" p. 7) can be given in a way that does not succumb to the Indefinite-Value Objection. However, the price to be paid to take this route seems to us too high, especially considering that there is a more conservative option, ours, which delivers the same riches. Torza characterizes the notion of non-defectiveness in terms of lack of expressions that are either semantically indeterminate (such as "bald") or irreferential (such as "Vulcan"), but this just moves the issue a level up. What makes an expression semantically indeterminate (or a singular term irreferential) is the relation in which it stands to its target - that is the actual world, in the case at issue. The representational capacities of a language are never defective in some absolute sense, but always with respect to the intended target of the representation. Therefore, without an object level account that can beef up 
the notion of non-defectiveness, Torza's account - insofar as it is seen as an attempt to reconcile the meta-level approach with quantum mechanics - is explanatorily less efficacious than ours, which does not rely on the intelligibility of an object level account of metaphysical indeterminacy.

Unlike PMS, both Darby \& Pickup's and Torza's variants of MS depart significantly from the fundamental idea of supervaluationism, that of capturing indeterminacy by relying on classical and complete precisifications. A further point is that they focus only on the Indefinite-Value Objection and do not consider the Interference-Pattern and the Statistics Objections. Their responses to the challenge from QI is hence incomplete as it stands. We will discuss the remaining two objections from the perspective of PMS in the next subsection.

\subsection{Plural MS and Quantum Mechanics}

Recall from $\S 4$ that according to PMS, in cases of QI ersatz worlds count as precisifications only together and as a whole. Each ersatz world can in such a case be said to on its own determinately fail to misrepresent reality. In cases of QI however, a set of ersatz worlds can jointly correctly represent reality, even though none of them alone can. If we stick with the second interpretation of $R_{p p}$, this gives us a glutty view of indeterminacy, whereas MS, or a version of PMS incorporating the more conservative first interpretation of $R_{p p}$ gives us a gappy view. According to MS, indeterminacy is unsettledness between precise ersatz worlds that do not determinately misrepresent reality; according to PMS with the second interpretation of $R_{p p}$, in some cases of indeterminacy — including QI-reality is represented by an irreducible compresence of many precise ersatz worlds. Metaphorically, for MS, if there is QI the world does not settle which ersatz world is actualized; for PMS, if there is QI the world settles that more than one ersatz world is actualized.

Let us now go back to the example given in $\S 5.1$ of an electron being in state $S=\mid \uparrow z>$, with the corresponding indeterminacy of $y$-spin and $x$-spin. While MS would account for such indeterminacy in terms of the various precisifications taken separately, PMS rather takes the following set of ersatz worlds to plurally represent the indeterminacy:

$w 1:\left(S_{\mathrm{z} \text {-spin }}=\uparrow\right) \&\left(S_{\mathrm{y} \text {-spin }}=\downarrow\right) \&\left(S_{\mathrm{x} \text {-spin }}=\downarrow\right)$

$w 2:\left(S_{\text {z-spin }}=\uparrow\right) \&\left(S_{\text {y-spin }}=\uparrow\right) \&\left(S_{\text {x-spin }}=\downarrow\right)$ 


$$
\begin{aligned}
& \text { w3: }\left(S_{\mathrm{z} \text {-spin }}=\uparrow\right) \&\left(S_{\mathrm{y} \text { spin }}=\downarrow\right) \&\left(S_{\mathrm{x} \text {-spin }}=\uparrow\right) \\
& w 4:\left(S_{\mathrm{z} \text {-spin }}=\uparrow\right) \&\left(S_{\mathrm{y} \text {-spin }}=\uparrow\right) \&\left(S_{\mathrm{x} \text {-spin }}=\uparrow\right)
\end{aligned}
$$

So PMS relies on the same ersatz worlds to model this case of QI as MS. As we would have expected, these four ersatz worlds do not disagree regarding $\left(S_{z \text {-spin }}=\uparrow\right)$, since the relevant model reflects the physical fact that there is no indeterminacy about the system's $z$-spin. The ersatz worlds however disagree as to whether the system is up or down in both $x$ and $y$, reflecting the indeterminacy of these properties. The difference to MS lies in the role played by the four ersatz worlds in the model. MS requires each of $w 1-w 4$ on its own to not definitely misrepresent the actual world, in order for each to qualify as an admissible precisification. Since none of the four worlds does this for the reasons given in $\$ 5.1$, the model cannot accurately represent the indeterminacy of the system with respect to the two observables $S_{\mathrm{x} \text {-spin }}$ and $S_{\mathrm{y} \text {-spin }}$.

The fact that none of the four ersatz worlds alone qualifies as an admissible precisification of the indeterminate portion of reality we are intending to capture provides the starting point for the PMS-based interpretation of the model. None of $w 1-w 4$ on its own qualifies as an admissible precisification in the standard, singular sense. But taken together they manage to plurally represent the indeterminacy of reality in an accurate way, since they provide us with the set of worlds of an admissible PMS-model of reality. With this general explanatory scheme in mind, let us see how PMS addresses the three objections based on quantum mechanics.

\subsubsection{The Indefinite-Value Objection and PMS}

Recall that the problem with MS was that the precisifications were individual fully precise possible worlds. But since QM does not allow us to assign precise values to each property, it followed that none of the precisification was a candidate for representing reality, since they all determinately misrepresents reality. As we saw in $\$ 5.2$, one way to circumvent the objection is to claim that precisifications need not be precise (as in Torza, and Darby \& Pickup). PMS takes a different path, and insists that while ersatz worlds are still fully precise and nomologically classical (i.e., they do not show quantum behavior), none of them is an admissible precisification, since only taken collectively they can represent QI. PMS does not take single classical worlds to be admissible precisification in such 
cases. Rather, being an admissible precisification in PMS is a genuine plural notion that applies to a plurality of worlds. And notice that it seems plausible to assume that representational entities are nomologically classical, because this is more often than not the way we represent things.

One could object to this move by noticing that simply by multiplying classical representations we do not, in principle, get to understand non-classical scenarios. Although it is made in the context of standard MS, the following remark by Calosi and Wilson (2019) makes this objection even more pressing:

Taking precisifications to be ones in which classical laws are operative violates supervaluationist constraints on admissible precisifications-namely, that precisifications cannot be determinately incompatible with (cannot determinately misrepresent) the actual world. In particular, the true claim that 'the position and momentum of a system cannot be jointly fully precise' is determinately true if the actual world is, as we are assuming, a quantum world; but classical worlds in which every system has determinate position and momentum will be worlds in which this claim is false, not true; hence any such world would fail to be an admissible precisification. (p. 18)

The idea is that in order to represent a quantum world, an ersatz world has to contain quantum laws as propositions. But if it does, it cannot also contain propositions that are in contradiction with those laws. For example, an ersatz world $w$ cannot contain the proposition that $x$-spin, $y$-spin, and $z$-spin cannot be jointly fully precise (which follows from the laws), and the proposition that $x$-spin, $y$-spin, and $z$-spin are assigned jointly a precise value, on pain of contradiction. If the latter proposition is dropped, the ersatz world is not after all fully precise, and thus it fails to possibly be an adequate representation of the actual world; if the former is, it looks like the ersatz world is determinately misrepresenting reality, since it is incoherent.

Although we believe this is a fair objection against standard MS with its singular admissibility-relation, we do not think that it applies to PMS. The theoretical role allocated to ersatz worlds in PMS is not to individually be a representation of the actual world that is adequate. Therefore, nothing bad for our theory follows if we stipulate that they do not contain the laws of quantum mechanics, and thus each of them, individually, cannot be an adequate representation of the actual world, when it comes to QI. The very idea of the theory is that single ersatz worlds are not to be understood as 
adequate representations of reality, but that pluralities of ersatz worlds taken together are. Hence, the complaint that laws have to show up in any adequate representation of the world is misplaced with respect to individual ersatz worlds. ${ }^{28}$

\subsubsection{The Statistics Objection and Interference-Pattern Objection}

Recall that according to Calosi and Wilson (2019) MS is not able to account for the difference between superpositions of different statistical weights (like $50 \% / 50 \%$ and $75 \% / 25 \%$ ). There are in fact several ways to represent these weights in PMS. We will briefly mention two.

First, we can recover the statistics through the number of ersatz worlds in a model by defining an appropriate metric. To do this, we include a suitable number of ersatz worlds in the set of worlds $W$ of our models, such that among these worlds, for each state $s$, the ratio between the number of worlds according to which $s$ obtains to the total number of worlds gives us the statistical weight assigned to $s$. E.g., the total number of ersatz worlds in $W$ of the model could simply be 2 when the probability distribution we have to recover is $50 \% / 50 \%$ between two distinct states (i.e. we would not have to inflate the number of worlds compared to a "regular" model which is not specifically constructed to reflect the probabilities of the relevant states), or 4 ersatz worlds when it is $25 \% / 75 \%$ also between two distinct states, and so on. To implement this response, $R_{p p}$ would have to be modified accordingly.

A second strategy relies not on the number of worlds in an admissible model, but rather assigns the statistical weight directly to the ersatz worlds

${ }^{28}$ A natural question to ask is where we find the laws in PMS. A way to address this issue is by accepting a bifurcated treatment of laws of nature, which draws on a well-established distinction between two kinds of physical laws, namely the dynamical and kinematical ones. Maudlin (2007) for instance, speaks of FLOTEs - Fundamental Laws of Temporal Evolution - to refer to the former, and adjunct principles to refer to the latter. We could suppose that the only laws that need to be recovered are the kinematical ones, since these are the laws that generate indeterminacy (namely, those that we cannot have as sentences within the ersatz worlds). The kinematics could potentially be recovered at the meta-level as a set of constraints across the possibilities; after all, this is precisely what the kinematics is. Such a view, though admittedly underdeveloped, does not seem to conflict irremediably with our understanding of physical laws. Be that as it may, we stress that this issue is independent from whether or not the ersatz worlds must contain all the laws as propositions in PMS; as we argued, there is no reason why they should. 
representing the states in the model. One way of implementing this second strategy is to turn $R_{p p}$ into a relation which holds between a universe and a set of tuples each containing first, one ersatz world and, second, a number suitable to capture the relevant probabilities. This second option would require a more radical modification of the model theory of PMS.

It has to be noticed that broadly similar strategies are adopted independently of considerations about QI in the context of the Many-World interpretation of QM. The Many-Minds interpretation of Quantum Mechanics is a case in point. ${ }^{29}$ However, we should point out that in case of PMS, increasing the number of ersatz worlds or modifying the models in the suggested way is arguably less costly, since the entities that are multiplied are still only abstract entities posited in the context of a theory of metaphysical indeterminacy, i.e. not the sort of entities which add to one's fundamental ontology.

Finally, turning to the Interference-Pattern Objection, recall that the problem is how we can explain interference effects, like for example in the double slit experiment. Ersatz worlds cannot interact with each other, so how does the interference pattern emerge? While we have to admit that interference effects cannot be fully explained by PMS, we shall also notice that other accounts of metaphysical indeterminacy have the same problem here, including of course other theories which rely on ersatz worlds, as well as Lewisian "real" worlds - should a theory relying on them ever be proposed. Notably, even Calosi \& Wilson's (2019) approach faces the same difficulty, although they are the ones who proposed the objection in the first place. Their view can either be implemented in gappy terms (no determinate of the relevant determinable is instantiated), or in glutty terms (more than one is instantiated). Of course, as they too recognize explicitly, a gappy implementation would suffer from the very same problem; if there are no determinate properties (e.g. 'passing through slit-1', and 'passing through slit-2'), how can the system generate the fringes of interference? For this reason, Calosi and Wilson (2019) suggest that a glutty approach is to be preferred. However, the glutty version of their approach is still underdeveloped. They suggest that each of the determinates forming the glut is instantiated to a non-zero degree, providing "an occurrent physical basis for the interference effect" (Calosi \& Wilson 2019, p. 24). But as of now there is no cogent explanation of how properties can be instantiated in

\footnotetext{
${ }^{29}$ See e.g. Albert \& Loewer 1988. Thanks to Jonathan Schaffer here.
} 
degrees, and even if there were, it would still have to be shown how this degree-ontology can allow for causal interaction of the kind we see in the double-slit experiment.

Quantum interference is still among the main mysteries of quantum mechanics - the main one, according to Feynman (1965) — and arguably it will remain so independently of which account of quantum indeterminacy turns out to be the best one.

\section{Conclusion}

Value indefiniteness in QM arguably prompts us to accept that the world itself, rather than our representations of it can be indeterminate. Cases of value indefiniteness have also been identified as the Achilles heel of supervaluationism, a theory which has otherwise established itself as perhaps the standard theory of metaphysical indeterminacy. PMS provides us with a general theory of metaphysical indeterminacy which meets the crucial challenge posed by Quantum Mechanics.

\section{Acknowledgements}

We would like to thank two anonymous referees for their comments and suggestions. We also thank Claudio Calosi, Jonathan Schaffer, Jonathan Tallant, Alessandro Torza, the participants of a session of the eidos-seminar at the University of Geneva, the audience at the 4th Society for the Metaphysics of Science Conference, and the participants of the Quantum Indeterminacy Workshop at Dartmouth University, for useful comments on previous versions of this manuscript. C. M.'s work was supported by the Foundational Questions Institute Fund (Grant number FQXi-IAF19-05 and FQXi-IAF19-01. R. M.'s work on the paper was made possible by the Schweizerischer Nationalfonds zur Förderung der Wissenschaftlichen Forschung (Project 'Identity in Cognitive Science, Quantum Mechanics, and Metaphysics', grant number: 185435, PI: Kevin Mulligan). G. T. wishes to acknowledge financial support from the project CHRONOS (PID2019-108762GB-I00) of the Spanish Ministry of Science and Innovation, and the Department of Philosophy "Piero Martinetti" of the University of Milan under the Project "Departments of Excellence 2018-2022" awarded by the Ministry of Education, University and Research (MIUR). 


\section{References}

- Akiba, K. (2000a). Vagueness as a modality. Philosophical Quarterly, 50(200): 359-370.

- Akiba, K. (2000b). Indefiniteness of mathematical objects. Philosophia Mathematica, 8(1): 26-46.

- Akiba, K. (2004). Vagueness in the world. Nô̂s, 38(3): 407-429.

- Akiba, K. \& Abasnezhad, A. (eds.) (2014). Vague Objects and Vague Identity: New Essays on Ontic Vagueness. Springer.

- Albert, D. and Loewer, B. (1988). Interpreting the Many Worlds Interpretation. Synthese, 77: 195-213.

- Asher, N. Dever, J. \& Pappas, C. (2009). Supervaluations debugged. Mind, 118(472): 901-933.

- Barnes, E. (2010). Ontic vagueness: A guide for the perplexed. Nô̂s, 44(4): 601-627.

- Barnes, E. (2013). Metaphysically indeterminate existence. Philosophical Studies, 166(3): 495-510.

- Barnes, E. \& Cameron, R. (2008). The open future: bivalence, determinism and ontology. Philosophical Studies, 146 (2):291-309.

- Barnes, E. and Williams, J. R. G. (2011). A theory of metaphysical indeterminacy. Oxford Studies in Metaphysics, 6: 103-148.

- Barwise, J. \& Perry, J. (1983). Situations and Attitudes. MIT Press.

- Bokulich, A. (2014). Metaphysical Indeterminacy, Properties, and Quantum Theory. Res Philosophica, 91(3): 449-475.

- Cabello, A. Estebaranz, J. \& Garcì-Alcaine, G. (1996). Bell-Kochen-Specker Theorem: A Proof with 18 vectors, Physics Letters A, 212: 183-87.

- Calosi, C. \& Mariani, C. (2020). Quantum Relational Indeterminacy. Studies in History and Philosophy of Science Part B: Studies in History and Philosophy of Modern Physics, 71: 158-169.

- Calosi, C. \& Mariani, C. (2021). Quantum Indeterminacy. Philosophy Compass. Doi:10.1111/phc3.12731.

- Calosi, C. \& Wilson, J. (2019). Quantum metaphysical indeterminacy. Philosophical Studies, 176(10): 2599-2627.

- Chalmers, D. J. (2006). The Foundations of Two-Dimensional Semantics. In Garcia-Carpintero M. \& Macia J. (eds.), Two-Dimensional Semantics: Foundations and Applications. Oxford University Press: 55-140. 
- Chen, E. K. (2020). Nomic Vagueness. arXiv:2006.05298[physics.hist-ph].

- Corti, A. (Forthcoming). Yet Again, Quantum Indeterminacy is Not Worldly Indecision. Synthese. Doi:10.1007/s11229-021-03039-1.

- Darby, G. (2010). Quantum Mechanics and Metaphysical Indeterminacy. Australasian Journal of Philosophy, 88(2): 227-245.

- Darby, G., \& Pickup, M. (2019). Modelling Deep Indeterminacy. Synthese.

- Evans, G. (1978). Can There Be Vague Objects? Analysis, 38(4), 208.

- Feynman, R. (1965). The Character of Physical Law. BBC Publications.

- Field, H. (1994). Are Our Logical and Mathematical Concepts Highly Indeterminate? Midwest Studies in Philosophy, 19(1): 391-429.

- Fine, K. (1975). Vagueness, truth and logic. Synthese, 30(3-4): 265-300.

- Fraassen, Bas C. van. 1966. Singular Terms, Truth Value Gaps and Free Logic. Journal of Philosophy 63 (17): 481-95.

- Glick, D. (2017). Against quantum indeterminacy. Thought, 6(3): 204-213.

- Heisenberg, W. (2007). Physics and Philosophy: The Revolution in Modern Science. HarperCollins.

- Keefe, R. (2000). Theories of Vagueness. Cambridge University Press.

- Kochen, S. \& Specker, E. (1967). The Problem of Hidden Variables in Quantum Mechanics. Journal of Mathematics and Mechanics, 17: 59-87.

- Lewis, D. K. (1986). On the Plurality of Worlds. Wiley-Blackwell.

- Maudlin, T. (1995). Three measurement problems. Topoi, 14: 7-15.

- Maudlin, T. (2017). The Metaphysics Within Physics. Springer.

- Miller, E. (2013). Quantum Entanglement, Bohmian Mechanics, and Humean Supervenience. Australasian Journal of Philosophy, 92(3): 567-583.

- Oliver, A. \& Smiley, T. (2016). Plural Logic. 2nd edition. Oxford University Press UK.

- Rosen, G. \& Smith, N. J. J. (2004). Worldly indeterminacy: A rough guide. Australasian Journal of Philosophy, 82(1): 185 - 198.

- Rovelli, C. (1996). Relational Quantum Mechanics. International Journal of Theoretical Physics, 35(8): 1637-1678. 
- Schrödinger, E. (1935). Die gegenwärtige Situation in der Quantenmechanik. Die Naturwissenschaften, 23: 807-812, 823-828, 844-849.

- Skow, B. (2010). Deep metaphysical indeterminacy. Philosophical Quarterly, 60(241): 851-858.

- Thomason, R. H. (1970). Indeterminist time and truth-value gaps. Theoria, 36(3): 264-281.

- Torza, A. (2020). Quantum metaphysical indeterminacy and worldly incompleteness. Synthese, 197(10): 4251-4264.

- Tye, M. (1990). Vague Objects. Mind, 99: 535-557.

- Varzi, A. C. (2007). Supervaluationism and Its Logics. Mind, 116(463): 633-676.

- Varzi, A. C. (2020). Indeterminate Identities, Supervaluationism, and Quantifiers. Analytic Philosophy, 61 (3): 218-235.

- Wallace, D. (2016). What is Orthodox Quantum Mechanics? arXiv:1604.05973v1[quant-ph].

- Williams, J. R. G. (2008). Multiple actualities and ontically vague identity. Philosophical Quarterly, 58(230): 134-54.

- Williamson, T. (1994). Vagueness. Routledge.

- Wilson, J. M. (2013). A Determinable-Based Account of Metaphysical Indeterminacy. Inquiry: An Interdisciplinary Journal of Philosophy, 56(4): 359-385. 